\title{
Modeling Degranulation with Liposomes: Effect of Lipid Composition on Membrane Fusion
}

\author{
T.G. Brock ${ }^{1}$, K. Nagaprakash ${ }^{1}$, D.I. Margolis ${ }^{1}$, J.E. Smolen ${ }^{1,2}$ \\ ${ }^{1}$ Department of Pediatrics, University of Michigan Medical Center, Room 7510C MSRB I, Box 0684, Ann Arbor, Michigan 48109-0684 \\ ${ }^{2}$ Department of Pathology, University of Michigan Medical Center, Ann Arbor, Michigan 48109-0684
}

Received: 14 December 1993/Revised: 5 April 1994

\begin{abstract}
Degranulation involves the regulated fusion of granule membrane with plasma membrane. To study the role of lipid composition in degranulation, large unilamellar vesicles (LUVs) of increasing complexity in lipid compositions were constructed and tested for $\mathrm{Ca}^{2+}$ mediated lipid and contents mixing. Lipid-mixing rates of LUVs composed of phosphatidylethanolamine (PE) and phosphatidylserine (PS) were strongly decreased by the addition of either phosphatidylcholine (PC) or sphingomyelin (SM), while phosphatidylinositol (PI) had little effect. "Complex" LUVs of PC:PE:SM:PI:PS (24:27:20:16:13, designed to emulate neutrophil plasma membranes) also showed very low rates of both lipid mixing and contents mixing. The addition of cholesterol significantly lowered the $\mathrm{Ca}^{2+}$ threshold for contents mixing and increased the maximum rates of both lipid and contents mixing in a dose-dependent manner. Membrane remodeling, which occurs in neutrophil plasma membranes upon stimulation, was simulated by incorporating low levels of phosphatidic acid (PA) or a diacylglycerol (DAG) into complex LUVs containing $50 \%$ cholesterol. The addition of PA both lowered the $\mathrm{Ca}^{2+}$ threshold and increased the rate of contents mixing in a dose-dependent manner, while the DAG had no significant effect. The interaction of dissimilar LUVs was also examined. Contents-mixing rates of LUVs of two different cholesterol contents were intermediate between the rates observed for the LUVs of identical composition. Thus, cholesterol needed to be present in only one fusing partner to enhance fusion. However, for PA to stimulate fusion, it had to be present in both sets of LUVs. These results suggest that the rate of degranulation may be increased by a rise in the cholesterol
\end{abstract}

Correspondence to: J.E. Smolen level of either the inner face of the plasma membrane or the outer face of the granule membrane. Further, the production of PA can promote fusion, and hence degranulation, whereas the subsequent conversion of PA to DAG may reverse this promotional effect.

Key words: Calcium - Cholesterol - Liposomes Membrane fusion - Phosphatidic acid

\section{Introduction}

Degranulation involves the fusion of granule membrane to plasma membrane. Fusion, and hence degranulation, does not occur in resting neutrophils but proceeds rapidly following the appropriate stimulus. It is unclear what role, if any, lipid composition plays in controlling fusion during degranulation. However, membrane remodeling (e.g., through the action of various phospholipases) is known to be an integral part of a number of signaling pathways that lead to exocytosis (Smolen \& Shohet, 1974; Serhan et al., 1982; Agwu et al., 1989; Santini et al., 1990; Cockcroft, 1992). It is not known how membrane remodeling might mediate membrane fusion during degranulation.

The use of model membrane systems using one to three phospholipids has proven to be a powerful approach for studying the effect of individual lipids on fusion. However, few studies have examined the fusion properties of liposomes prepared with complex assemblages of phospholipids, as are found in biological membranes. Furthermore, the effect of cholesterol on the fusion of complex assemblages is unclear. In simpler model systems, cholesterol can inhibit (Papahadjopoulos et al., 1974; Breisblatt \& Ohki, 1976; Chaudhury \& Ohki, 1981; Connor, Yatvin \& Huang, 1984), enhance (Evans \& Needham, 1986; Rand \& Parsegian, 
1986) or be required (Kielian \& Helenius, 1984; Nieva, Goni \& Alonso, 1989) for fusion.

The present study details the fusion characteristics of liposomes designed to emulate the plasma membranes of neutrophils in composition. Following available analyses (Smolen \& Shohet, 1974; Cockcroft, 1984; Diez, Balsinde \& Mollinedo, 1990), complex liposomes contained predominantly phosphatidylcholine (PC) and phosphatidylethanolamine (PE), with significant amounts of sphingomyelin (SM), phosphatidylinositol (PI) and phosphatidylserine (PS). Membrane remodeling is known to occur in neutrophils (Smolen \& Shohet, 1974; Diez et al., 1990; Cockcroft, 1992), and so the effects of low levels of phosphatidic acid (PA) and a diacylglycerol (DAG) on fusion have been tested. Furthermore, increasing cytoplasmic $\mathrm{Ca}^{2+}$ is central to neutrophil degranulation (Smolen \& Sandborg, 1990; Smolen, 1992). Consequently, we have examined fusion as induced by $\mathrm{Ca}^{2+}$, although membrane fusion during degranulation undoubtedly involves more factors than $\mathrm{Ca}^{2+}$ alone (Düzgünes et al., 1987; Papahadjopoulos, Nir \& Düzgünes, 1990).

\section{Materials and Methods}

\section{Materials}

Egg PC, PA from egg PC, egg PE, liver PI, bovine brain PS, and egg SM were purchased from Avanti Polar Lipids (Birmingham, AL). Octadecyl rhodamine (R18), p-xylene-bis-pyridinium bromide (DPX), 8aminonaphthalene-1,3,6-trisulfonic acid (ANTS) were purchased from Molecular Probes (Eugene, OR). 1,2-dioctanoyl-sn-glycerol $\left(\mathrm{DiC}_{8}\right)$ was purchased from both Avanti and Sigma. All other chemicals were from Sigma.

\section{LIPOSOME PREPARATION}

Liposomes were composed of various combinations of PC, PE, SM, PI, and PS and cholesterol. These combinations were systematically related to achieving an ultimate ratio of $24: 27: 20: 16: 13$ PC:PE:SM:PI:PS. Any liposomes prepared with these five phospholipids in this ratio were designated "complex" liposomes, for brevity. LUVs were used for all assays and were prepared as described previously (Francis et al., 1992). Briefly, vesicle components were prepared and mixed in chloroform, dried in a near-vacuum at $37^{\circ} \mathrm{C}$ under argon, then resuspended in buffer. Buffers used were (in mM): KHEN (130 KCl, 30 HEPES, 1 EGTA, $10 \mathrm{NaCl}$, pH 7.0), 25 ANTS (in 19.5 $\mathrm{KCl}, 30$ HEPES, pH 7.0) or 117 DPX (in 30 HEPES, pH 7.0). Samples were frozen $\left(-70^{\circ} \mathrm{C}\right)$ thawed $\left(40^{\circ} \mathrm{C}\right)$ seven times, then extruded seven times through two $100 \mathrm{~nm}$ polycarbonate filters, using an Extruder (Lipex Biomembranes, Vancouver, British Columbia, Canada). Unincorporated materials were removed by gel filtration with Sephadex G-75. The concentration of liposomes in the pooled opaque fractions was evaluated by phosphorus determination (Morrison, 1964).

\section{Diacylglycerol Micelle Preparation}

$\mathrm{DiC}_{8}$ in chloroform was dried under a stream of nitrogen, resuspended in dimethylsulfoxide and diluted 20 -fold in KHEN buffer. The resulting opaque solution was sonicated for $30 \mathrm{sec}$ to completely disperse the diacylglycerol.

\section{LiPosome AgGREgation AsSAY}

Aggregation was assayed according to Meers et al. (1987), with the following modifications. LUVs ( $370 \mu \mathrm{M}$ lipid) were suspended in KHEN buffer with constant stirring. Changes in absorbance at 450 nm were continuously recorded by spectrophotometer (model DU-8, Beckman Instruments).

\section{LIPID-MIXING ASSAY}

The transfer of phospholipid between LUVs was assayed by recording the increase in rhodamine (R18) fluorescence (relief of selfquenching) due to R 18 dilution (Francis et al., 1990). LUVs labeled with $2 \mathrm{~mol} \%$ R 18 were stirred with unlabeled LUVs at a ratio of $1: 4$ $\left(100 \mu \mathrm{M}\right.$ total lipid) at $37^{\circ} \mathrm{C}$. Lipid mixing was evaluated as the initial rate of change in fluorescence, occurring over the first $15 \mathrm{sec}$, and recalculated as the percent maximum change per minute. Maximum change was defined as the change in fluorescence produced by the addition of $0.1 \%$ Triton X-100 to R18-labeled LUVs.

\section{CONTENTS-MIXING ASSAY}

The mixing of aqueous contents between LUVs was evaluated as the decrease in fluorescence from ANTS due to quenching by DPX, as described previously (Francis et al., 1992), ANTS-containing LUVs were stirred with DPX-containing LUVs at a ratio of 1:1 (370 $\mu \mathrm{M}$ total lipid) at $37^{\circ} \mathrm{C}$. Contents mixing was evaluated as the initial rate of change in fluorescence, occurring over the first $10 \mathrm{sec}$, and recalculated as the percent maximum change per minute. Contents leakage was also evaluated by assaying the quenching of ANTS fluorescence by $4.5 \mathrm{~mm}$ free DPX in parallel treatments. Maximum mixing was defined as the change in fluorescence produced by the addition of $4.5 \mathrm{mM}$ free DPX and $0.1 \%$ Triton X-100 to ANTS-containing LUVs.

\section{$\mathrm{Ca}^{2+}$ Threshold Determination}

The $\mathrm{Ca}^{2+}$ threshold for a given response, defined as the $\mathrm{Ca}^{2+}$ concentration above which a positive response would be attained, was determined by curve-fitting the three lowest positive response values, using Sigmaplot Scientific Graphing System, version 5.00, and then calculating the $\mathrm{Ca}^{2+}$ value corresponding with a response value of zero. Results are presented as the means $\pm \mathrm{SEM}$ of at least three separate experiments.

Aggregation, lipid mixing and contents mixing were initiated by the addition of various concentrations of $\mathrm{CaCl}_{2}$. Free $\mathrm{Ca}^{2+}$ concentration was determined by the method of Bers (1982). Fluorescence was measured by spectrofluorimeter (model 650-10S, Perkins-Elmer, Norwalk, CT) equipped with a temperature-regulated cell holder and a magnetic stirring device.

\section{ABBREVIATIONS}

ANTS, 8-aminonaphthalene-1,3,6-trisulfonic acid; $\mathrm{DiC}_{8}$, 1,2-dioctanoyl-sn-glycerol; DPX, $p$-xylene-bis-pyriđinium bromide; LUV, large unilamellar vesicle; PA, phosphatidic acid; PC, phosphatidylcholine; PE, phosphatidylethanolamine; PI, phosphatidylinositol; PS, phosphatidylserine; R18, octadecyl thodamine; SM, sphingomyelin. 


\section{Results}

\section{FUSION OF "Simple" Liposome MiXTURES}

In most membranes, the most abundant fusogenic phospholipids are PE and PS. LUVs composed solely of $\mathrm{PE} / \mathrm{PS}$ (molar ratio of 27:13) were found to fuse readily, with a $\mathrm{Ca}^{2+}$ threshold for lipid mixing of approximately $2 \mathrm{mM} \mathrm{Ca}^{2+}$ and a maximum initial rate of $18 \% /$ min achieved at a $\mathrm{Ca}^{2+}$ concentration of $4-5 \mathrm{mM}$ (Fig. 1). Other major phospholipids in cell membranes include PI, PC and SM. Liposomes composed of PE/PS/PI (27:13:16) showed comparable, although somewhat reduced, fusion properties to PE/PS LUVs. However, LUVs of either PE/PS/PC (27:13:24) or PE/PS/SM (27:13:20) showed greatly reduced lipidmixing capacities, with thresholds of $7.5-8 \mathrm{mM} \mathrm{Ca}^{2+}$ and maximum fusion rates of $<1 \% / \mathrm{min}$ (Fig. 1). Hence, $\mathrm{PI}$ appeared to have a neutral effect on fusion, while PC and SM were potent inhibitors.

\section{Aggregation and Fusion of "Complex" LIPOSOME MIXTURES}

To emulate the phospholipid composition of neutrophil plasma membranes, LUVs were prepared with the five major phospholipids found in these membranes: PC, PE, SM, PI and PS (molar ratios for PC:PE:SM:PI:PS of 24:27:20:16:13, after Diez et al. (1990)). Liposomes containing these five phospholipids at these ratios were designated "complex" LUVs. The first step in the fur sion process should be the apposition and aggregation of the participating liposomes. Using light scatter as a measure of aggregation, we found that the least amount of $\mathrm{Ca}^{2+}$ required to obtain a signal was approximately $5.8 \mathrm{~mm}$ (Fig. 2). If aggregation is an initial required step in fusion, then we would expect the fusion process to require the same or greater levels of $\mathrm{Ca}^{2+}$. As expected, the $\mathrm{Ca}^{2+}$ threshold for lipid mixing of complex LUVs was $9.3 \pm 0.3 \mathrm{mM} \mathrm{Ca}^{2+}$ (Fig. 3). Maximum initial rate of lipid mixing was $1.1 \pm 0.8 \% / \mathrm{min}$, attained at approximately $14 \mathrm{~mm} \mathrm{Ca}^{2+}$. Contents mixing for complex LUVs required more $\mathrm{Ca}^{2+}$, with a threshold of $10.0 \pm 0.2 \mathrm{mM} \mathrm{Ca}^{2+}$, and also reached a maximal rate of only $1.3 \pm 0.7 \% / \mathrm{min}$ (Fig. 4 ).

To further clarify the interactions between the liposomes, the reversibility of $\mathrm{Ca}^{2+}$-induced aggregation by EGTA was assessed. Changes in light scatter (presumably due to aggregation (Meers et al., 1987)) of complex LUVs were found to occur at $6.8 \pm 0.3 \mathrm{mM}$ $\mathrm{Ca}^{2+}$ (Fig. 5). When aggregation was initiated with 7.5 $\mathrm{mM} \mathrm{Ca}{ }^{2+}$ (i.e., below the level required for measurable contents mixing), the subsequent addition of EGTA reversed the light scattering signal, indicating aggregation without fusion. When aggregation was induced with

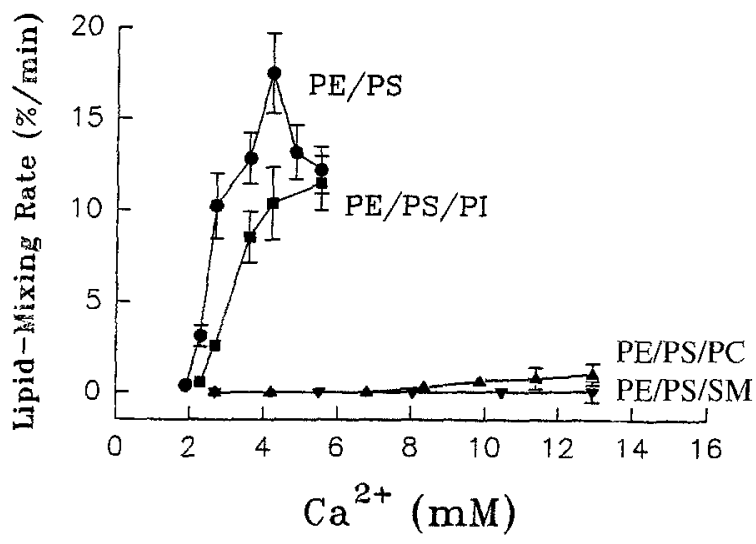

Fig. 1. The effects of PI, PC and SM incorporation on the initial rate of $\mathrm{Ca}^{2+}$-induced lipid mixing of PE/PS LUVs. Large unilamellar liposomes were prepared with PE/PS (27:13 molar ratio), PE/PS/PI (27:13:16), PE/PS/PC (27:13:24) or PE/PS/SM (27:13:20), with or without $2 \mathrm{~mol} \%$ rhodamine. Labeled $(20 \mu \mathrm{M})$ and unlabeled $(80 \mu \mathrm{M})$ liposomes were stirred at $37^{\circ} \mathrm{C}, \mathrm{Ca}^{2+}$ was added as a bolus to the desired concentration, and the initial rate of fusion was calculated from the resulting increase in fluorescence. Values are means \pm SEM. Representative experiment; $n=3$.

$11.3 \mathrm{mM} \mathrm{Ca}^{2+}$, the subsequent addition of EGTA did not reverse the light scattering signal, although it did prevent further aggregation. Therefore, these complex LUVs showed aggregation at 6-7 $\mathrm{mM} \mathrm{Ca}^{2+}$, whereas fusion occurred at higher $\mathrm{Ca}^{2+}$ levels.

\section{Cholesterol}

Cholesterol contents can differ greatly within different membranes from the same cell. For example, in neutrophils, cholesterol concentrations range from 27-33 mol \% for granule membranes to $40-50 \mathrm{~mol} \%$ for plasma membranes (Woodin \& Wieneke, 1966; Mason, Stossel \& Vaughan, 1972; Nachman, Hirsch \& Bagglioni, 1972; Werb \& Cohn, 1972). Furthermore, asymmetric distribution may result in cholesterol levels of as much as $90 \%$ in the inner face of the plasma membrane (Schroeder \& Nemecz, 1990). To determine the effect of cholesterol on fusion, complex LUVs were prepared with different concentrations of cholesterol. Both the threshold for aggregation and the initial rate of aggregation were independent of cholesterol level, remaining at $6-6.5 \mathrm{mM} \mathrm{Ca}^{2+}$ for all cholesterol concentrations (Fig. 2). However, the threshold for lipid mixing was reduced from $10 \mathrm{mM} \mathrm{Ca}^{2+}$ with no cholesterol to $8.5 \pm$ $0.4 \mathrm{mM} \mathrm{Ca}^{2+}$ with $50 \%$ cholesterol (Fig. 3). Also, increasing cholesterol concentrations increased the maximum rate of lipid mixing in a dose-dependent manner, from $1-2 \% / \mathrm{min}$ for cholesterol-free LUVs to $12 \pm$ $1.7 \% / \mathrm{min}$ for $50 \%$ cholesterol preparations. Contents mixing was also enhanced by cholesterol: increasing cholesterol to $50 \mathrm{~mol} \%$ resulted in a decrease of the 


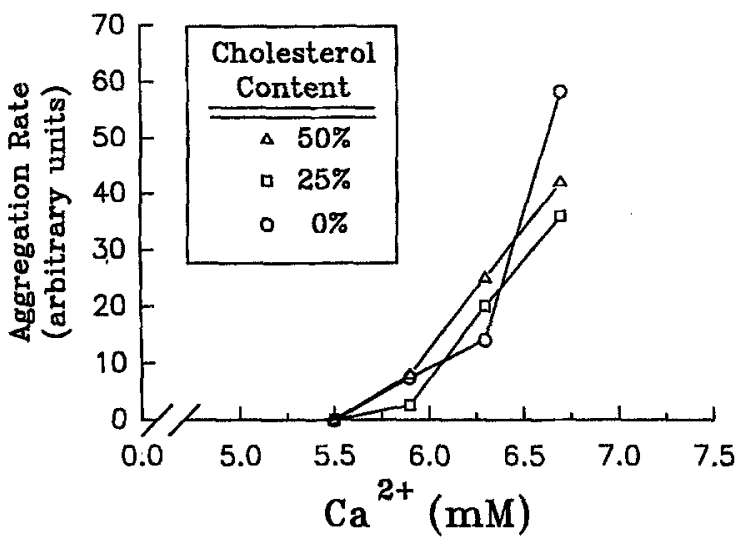

Fig. 2. The effect of cholesterol concentration on the initial rate of aggregation of complex LUVs. LUVS (PC:PE:SM:PI:PS of 24:27 $20: 16: 13$ ) were prepared with the indicated mol \% cholesterol, with the phospholipid ratios held constant. Aggregation of LUVs was assayed continuously at room temperature with continuous stirring. Representative experiment; $n=3$; standard errors were less than $20 \%$ of the mean.

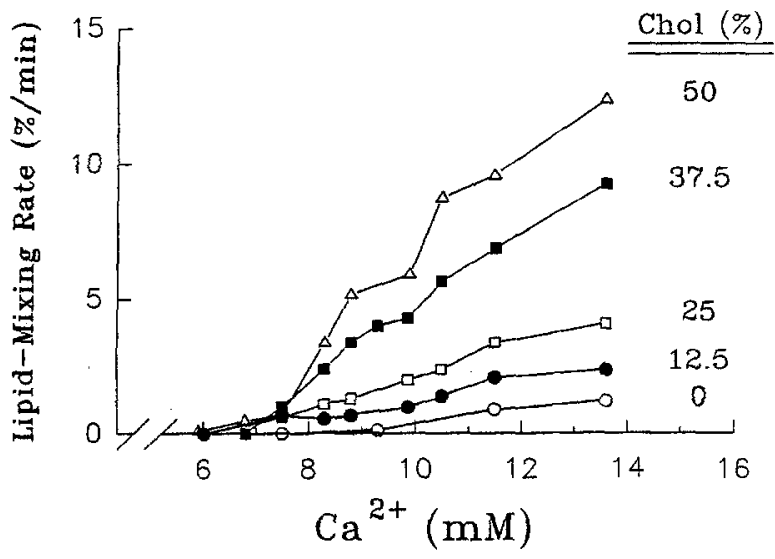

Fig. 3. The effect of cholesterol concentration on the initial rate of lipid mixing of complex LUVs. LUVs (PC:PE:SM:PI:PS of 24:27: 20:16:13) were prepared with the indicated mol \% cholesterol, with the phospholipid ratios held constant. The initial rate of lipid mixing was assayed at $37^{\circ} \mathrm{C}$. Representative experiment; $n=5$; standard errors were less than $20 \%$ of the mean.

threshold to $8.6 \pm 0.8 \mathrm{mM} \mathrm{Ca}^{2+}$. Much more strikingly, cholesterol increased the maximum rate of contents mixing, to $9.0 \pm 1.2 \% / \mathrm{min}$ at $9.9 \mathrm{mM} \mathrm{Ca}^{2+}$ (Fig. 4). Higher levels of $\mathrm{Ca}^{2+}$ further increased the rate of contents mixing of $50 \%$ cholesterol liposomes, reaching 53 $\pm 1.7 \% / \mathrm{min}$ at $13.6 \mathrm{mM} \mathrm{Ca}^{2+}$, but leakage also increased proportionately.

Because intracellular and extracellular concentrations of $\mathrm{Mg}^{2+}$ reach the low millimolar range, it was important to see what effect this divalent cation had on fusion of complex liposomes. We therefore determined the extent to which $\mathrm{Ca}^{2+}$ thresholds for aggregation and fusion were altered by increasing concentrations of

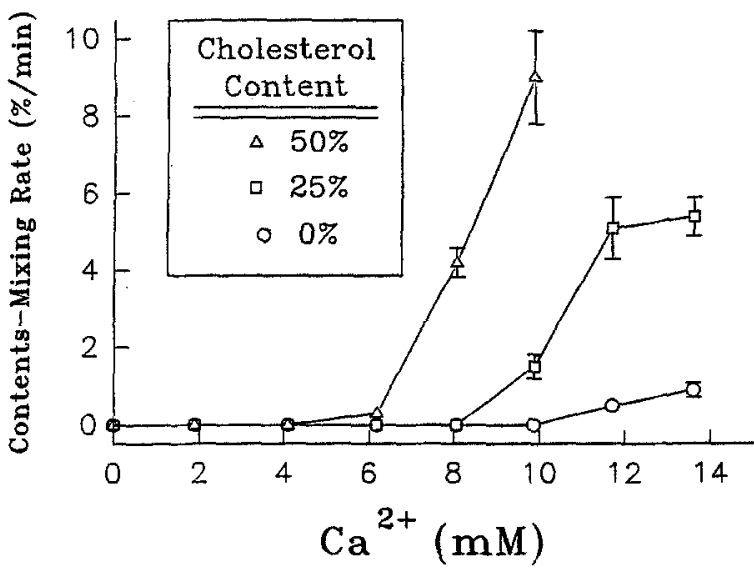

Fig. 4. The effect of cholesterol concentration on the initial rate of contents mixing of complex LUVs. The contents mixing of LUVs, prepared with the indicated mol \% cholesterol, was evaluated by the ANTS/DPX assay, using a 1:1 ratio of ANTS- to DPX-containing LUVs $(370 \mu \mathrm{M}$ total $)$ at $37^{\circ} \mathrm{C}$. Results indicate the initial rate of contents mixing following the addition of a bolus of $\mathrm{Ca}^{2+}$. Values are means \pm SEM. Representative experiment; $n=3$.

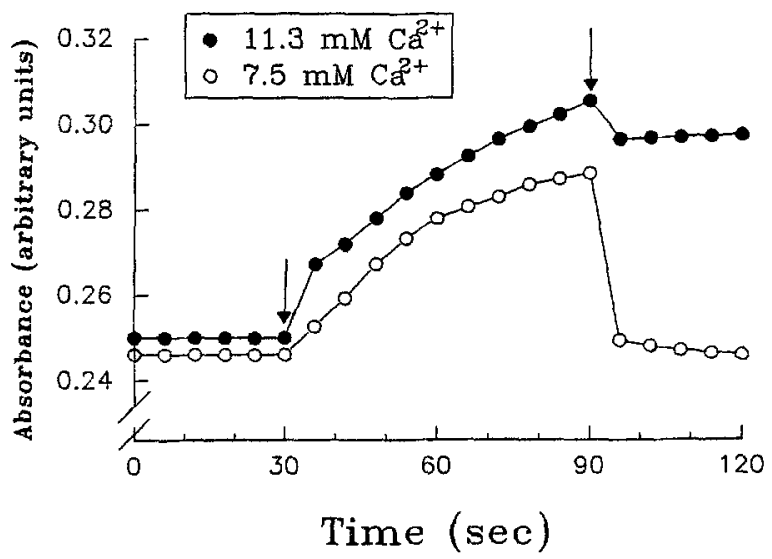

Fig. 5. Irreversible light scatter changes of complex LUVs lacking cholesterol. The absorbance of LUVs $(370 \mu \mathrm{M})$ at $450 \mathrm{~nm}$ was measured continuously after the addition of $\mathrm{Ca}^{2+}$ (first arrow; indicated concentration) and then the subsequent addition of $20 \mathrm{~mm}$ EGTA $1 \mathrm{~min}$ later (second arrow). Representative experiment; $n=5$.

$\mathrm{Mg}^{2+}$. For $50 \%$ cholesterol liposomes, the addition of millimolar levels of $\mathrm{Mg}^{2+}$ lowered the threshold for contents mixing, and $\mathrm{Mg}^{2+}$ alone at $14 \mathrm{~mm}$ could produce contents mixing (Table). Aggregation was even more sensitive, with $\mathrm{Mg}^{2+}$ being able to replace $\mathrm{Ca}^{2+}$ at concentrations of $10 \mathrm{~mm}$ and above. Thus, these two divalent cations cooperate in inducing aggregation and fusion of complex liposomes.

\section{Phosphatidic Acid AND $\mathrm{DiC}_{8}$}

PA and diacylglycerols appear transiently and locally in a variety of membranes, including neutrophil plasma 
Table. Effect of $\mathrm{Mg}^{2+}$ on $\mathrm{Ca}^{2+}$ thresholds for aggregation and fusion of complex liposomes

\begin{tabular}{lll}
\hline $\mathrm{Mg}^{2+}(\mathrm{mM})$ & \multicolumn{2}{l}{$\mathrm{Ca}^{2+}$ thresholds $(\mathrm{mM})$} \\
\cline { 2 - 3 } & Aggregation & Contents Mixing \\
\hline 0 & $9.3,8.1,6.8$ & $8.1,10,6.8$ \\
1.2 & $5.5,6.8$ & $\mathrm{ND}$ \\
2.5 & $5.5,6.8$ & 6.8 \\
5.0 & $4.1,5.5$ & 5.5 \\
7.5 & $2.6,4.1$ & 4.1 \\
10 & 0 & $2.5,1.0$ \\
14 & $\mathrm{ND}$ & 0
\end{tabular}

Complex liposomes containing 50\% cholesterol were prepared and $\mathrm{Ca}^{2+}$ thresholds for aggregation and contents-mixing fusion were determined as outlined in Materials and Methods and legends to Figs. 2 and 4 . These thresholds were determined in the presence of the indicated concentrations of $\mathrm{Mg}^{2+}$. From one to three experiments were performed for each condition, with each threshold determination being given above.

membranes, by a variety of mechanisms. To assess the effects of PA and diacylglycerol formation on fusion in membrane formulations, complex LUVs containing $50 \%$ cholesterol were prepared with $0-5 \mathrm{~mol} \% \mathrm{PA}$ or $0-5 \mathrm{~mol} \% \mathrm{DiC}_{8}$. Increasing the concentration of PA produced a decrease in the threshold for contents mixing, from $8.6 \mathrm{mM} \mathrm{Ca}^{2+}$ for PA-lacking LUVs to $5.8 \pm$ $0.6 \mathrm{mM} \mathrm{Ca}^{2+}$ for $5 \%$ PA preparations (Fig. 6). Furthermore, incorporation of $5 \mathrm{~mol} \% \mathrm{PA}$ also increased the maximal rate of fusion.

In contrast, the incorporation of up to $5 \% \mathrm{DiC}_{8}$ did not significantly change the contents-mixing characteristics (Fig. 7a). This result was obtained with $\mathrm{DiC}_{8}$ from two different suppliers. Similarly, the addition of $20 \mu \mathrm{M}$ $\mathrm{DiC}_{8}$ micelles to $100 \mu \mathrm{M} 50 \%$ cholesterol complex LUVs, prior to the addition of $\mathrm{Ca}^{2+}$, did not significantly enhance $\mathrm{Ca}^{2+}$-mediated LUV fusion (Fig. $7 b$ ).

\section{"Heterologous" Fusion}

Membrane fusion during degranulation involves two membranes that differ in composition. Consequently, we wished to see how fusion between liposomes of differing compositions compared with those of homologous composition. When two sets of LUVs differing in composition were tested for their requirements for fusing with each other (heterologous fusion), the results were typically intermediate between those for the corresponding identical partner pairings (homologous fusion). For example, when $12.5 \%$ cholesterol complex LUVs were tested with $50 \%$ cholesterol complex LUVs, the initial rates of contents mixing were typically less than those observed in 50\%/50\% homologous pairings and greater than those for $12.5 \% / 12.5 \%$ pairings

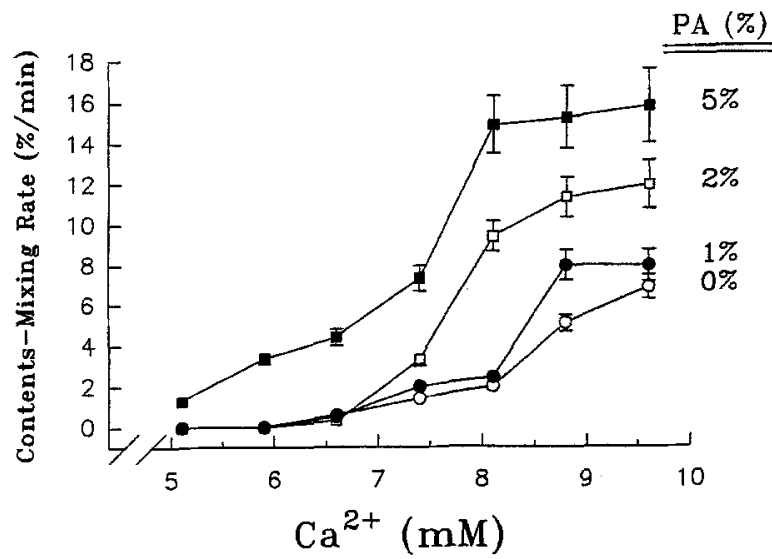

Fig. 6. The effect of PA incorporation on the initial rate of contents mixing of $50 \%$ cholesterol complex LUVs. Sets of liposomes were prepared with the indicated concentrations of PA, with either ANTS or DPX, and $\mathrm{Ca}^{2+}$-induced contents mixing was evaluated at $37^{\circ} \mathrm{C}$. Values are means \pm SEM. Representative experiment; $n=5$.

(Fig. 8). Similar patterns were found for several other heterologous pairings (data not shown).

However, not all cases of heterologous pairings gave results that were intermediate between the corresponding homologous pairings. Most notably, if $2 \% \mathrm{PA}$ was incorporated into only one partner of a $25 \% / 50 \%$ pairing, then fusion proceeded as though neither fusion partner contained PA (Fig. 9). In contrast, when both partners contained $2 \% \mathrm{PA}$, there were noticeable changes in both the threshold and the maximal rate of contents mixing.

\section{Discussion}

\section{"SIMPLE" LIPOSOMES}

The fusion of cell membranes, as in neutrophil degranulation, involves the interaction of two heterogeneous molecular ensembles. Our "complex" LUVs represented a very simplified membrane system that allowed a better understanding of the role of specific membrane components in the fusion process. One immediate conclusion is that both PC and SM drive the $\mathrm{Ca}^{2+}$ threshold up dramatically, whereas PI does not. Both PC and SM also act to significantly decrease the maximal rate of fusion. Previous studies have also shown that liposomes containing PC and SM are less fusogenic than liposomes constructed without these phospholipids (Düzgünes et al., 1981; Uster \& Deamer, 1981; Stamatotos \& Silvius, 1987). However, whereas PI incorporation is relatively neutral in effect on the fusion of $\mathrm{PE} / \mathrm{PS}$ liposomes, it is apparent that either PC or SM incorporation strongly inhibits $\mathrm{Ca}^{2+}$-mediated fusion. 
a

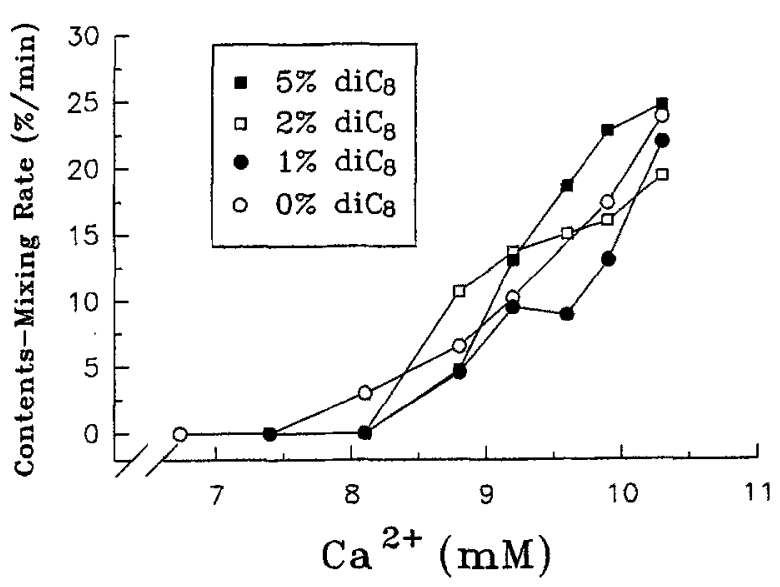

b

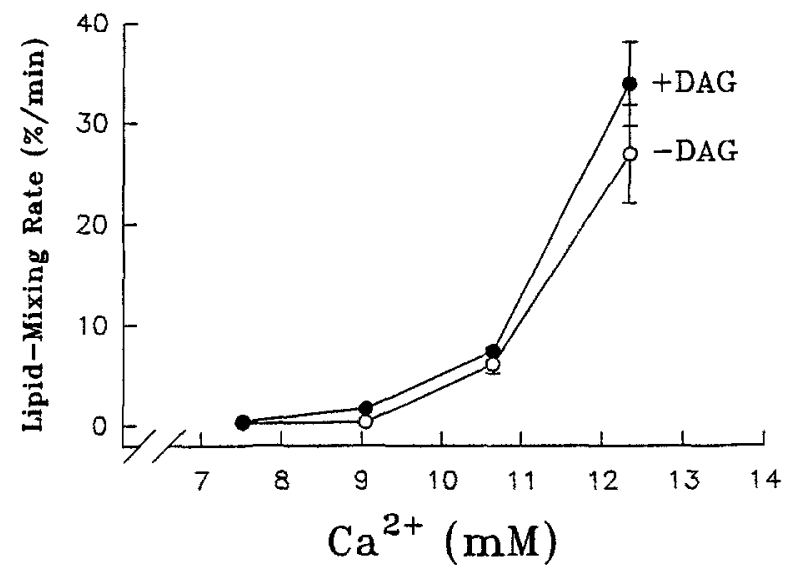

Fig. 7. The effect of $\mathrm{DiC}_{8}$ on the fusion of 50\% cholesterol complex LUVs. (a) The effect of $\mathrm{DiC}_{8}$ incorporation on contents mixing. LUVs were prepared with $\mathrm{DiC}_{8}$ at the indicated concentrations and assayed for contents mixing by the ANTS/DPX assay at $37^{\circ} \mathrm{C}$. Representative experiment; $n=4$; standard errors were less than $20 \%$ of the mean. $(b)$ The effect of exogenous $\mathrm{DiC}_{8}$ on lipid mixing. LUVs were prepared without $\mathrm{DiC}_{8}$. LUVs $(100 \mu \mathrm{M})$ were then stirred for $1 \mathrm{~min}$ with $\mathrm{DiC}_{8}$ micelles $(20 \mu \mathrm{M})$, and $\mathrm{Ca}^{2+}$ was added to induce fusion. Values are means \pm SEM. Representative experiment; $n=3$.

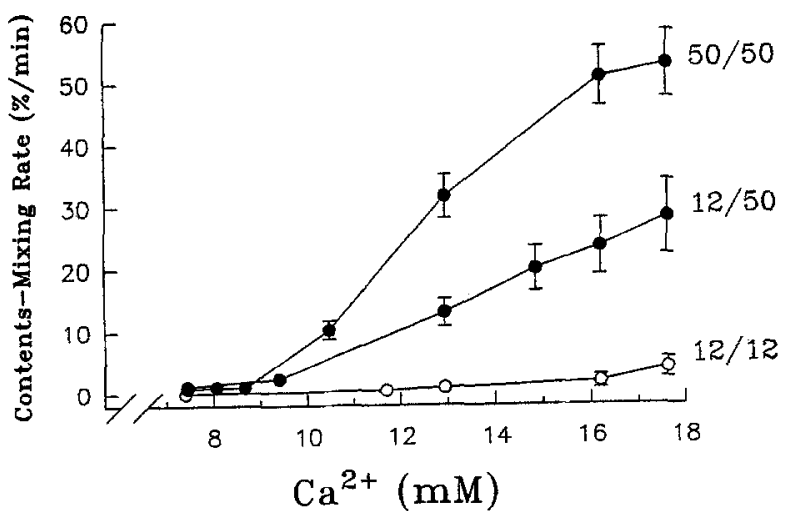

Fig. 8. A comparison of the characteristics of fusion between LUVs of difference $v s$. identical cholesterol content. Complex LUVs were prepared with either 12.5 or $50 \%$ cholesterol and assayed for contents mixing by the ANTS/DPX assay. Values are means \pm SEM. Representative experiment; $n=3$.

Similar findings were reported earlier by Sundler, Düzgünes and Papahadjopoulos (1981). Variation in the amount of PC or SM in cell membranes may be one effective way that cells could regulate the fusion capacity of that membrane.

\section{"COMPLEX" LIPOSOMES}

We ultimately wished to use liposomes that could serve as models for neutrophil membranes. Published analyses of neutrophil plasma membrane lipids (Smolen \& Shohet, 1974; Cockcroft, 1984) suggest PC/PE/ SM/PI/PS ratios of approximately 40/35/13/4/7, with

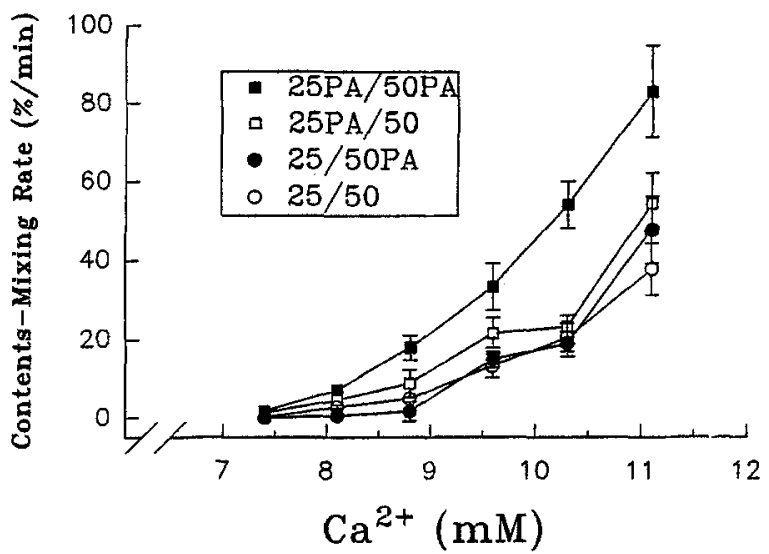

Fig. 9. The effect of $2 \mathrm{~mol} \% \mathrm{PA}$ incorporation into neither, one or both partners in a heterologous fusion assay. Complex LUVs were prepared with either 25 or $50 \%$ cholesterol, with or without PA, and assayed for contents mixing by the ANTS/DPX assay. Values are means \pm SEM. Representative experiment; $n=3$.

substantial variations. However, biological membranes are asymmetric with the cytoplasmic surfaces being much richer in PE and acidic phospholipids (Etemadi, 1980; Sessions \& Horwitz, 1983; Rand \& Parsegian, 1986; Verhoven, Schlegel \& Williamson, 1992). With this in mind, we enriched $\mathrm{PE}$ relative to $\mathrm{PC}$ and enhanced PI and PS concentrations. Our ultimate phospholipid ratios, designed to approximate the cytoplasmic surfaces of neutrophil membranes were 24:27: $20: 16: 13$. We chose as our model the results of Diez et al. (1990), who measured phospholipid contents in subcellular fractions (see Fig. 3 of that paper), including plasma membrane. 
These compositions admittedly provide only crude models for the endogenous neutrophil lipids:

(i) The fatty acid compositions have not been directly modeled. Our phospholipids were of natural origin, containing a spectrum of fatty acids, in the hope of partially addressing those concerns. Only perfect knowledge of the fatty acid composition of each phospholipid would allow us to address this adequately. The other alternative, making liposomes from extracted neutrophil lipids, was impractical due to yield.

(ii) Neutrophils contain a high content 1-O-alkyl linked phospholipids (Mueller et al., 1984), which we have not modeled. Unfortunately, we do not have practical sources for these types of lipids to adequately address this concern.

(iii) "Trace" components, such as polyphosphoinositides, may be crucial in fusion and degranulation (Eberhard et al., 1990; Horkovics-Kovats \& Traub, 1990). However, the composition of neutrophil membranes is not sufficiently known to account for such materials.

(iv) As noted above, biological membranes are asymmetric in composition (Etemadi, 1980; Sessions \& Horwitz, 1983; Rand \& Parsegian, 1986; Verhoven et al., 1992), and this asymmetry may be important in fusion (Eastman et al., 1992; Devaux et al., 1993). Creating asymmetry in model membranes is very difficult and such manipulations produce only crude changes at best (Eastman et al., 1992). Furthermore, the extent of asymmetry in neutrophil membranes is not known. To partially address this problem, we used a liposome preparation that was enriched in PE and acidic phospholipids.

(v) With these complex formulations, it was impossible to ensure that all the liposomes were identical in composition and properties.

These five limitations prevent us from modeling neutrophil membrane lipids with complete fidelity. These limitations must be remembered when interpreting our results. However, we are one of the few groups of investigators who have attempted to use "complex" lipid mixtures to more closely emulate biological compositions.

\section{Cholesterol}

In complex (PE/PC/SM/PI/PS) liposomes, cholesterol had clear effects: increasing cholesterol content increased the maximal rates of lipid and contents mixing, and it also decreased the $\mathrm{Ca}^{2+}$ requirement for contents mixing. In other studies, the effects of cholesterol on fusion have been inconsistent. One explanation, as suggested by Stamatotos and Silvius (1987), may be that cholesterol enables fusion in nonfusing liposome constructs, enhances fusion in poorly fusing ones (as it did here), and diminishes the fusion of rapidly fusing liposomes. Also, the positive effect of cholesterol on fusion may be masked by a delaying effect on aggregation, in certain types of liposomes. This pattern has been observed in PS-rich liposomes (Braun, Lelkes \& Nir, 1985; Bental et al., 1987), but does not apply to complex liposomes.

We also found that high concentrations of $\mathrm{Mg}^{2+}$ could assist $\mathrm{Ca}^{2+}$ for both aggregation and contentsmixing fusion (Table) when complex liposomes were used. $\mathrm{Mg}^{2+}$ ions are typically present in millimolar levels in the cytosol (Grubbs \& Maguire, 1987), and peaks of $\mathrm{Mg}^{2+}$ distribution are associated with the plasma membrane and granules in neutrophils (Raja et al., 1982). Millimolar levels of $\mathrm{Mg}^{2+}$ will significantly reduce the $\mathrm{Ca}^{2+}$ requirements for fusion in many systems (Düzgünes et al., 1987), including this one. If $\mathrm{Mg}^{2+}$ was raised sufficiently, this divalent cation alone was adequate to induce aggregation $\left(10 \mathrm{mM} \mathrm{Mg}^{2+}\right)$ or fusion (14 $\mathrm{mm} \mathrm{Mg}^{2+}$ ).

\section{PA AND $\mathrm{DiC}_{8}$}

As pointed out earlier, membrane remodeling may be an important step in enabling or enhancing fusion. That is, rapid restructuring may convert membranes from unwilling to willing fusion partners. On the basis of earlier studies, a primary candidate for a highly fusogenic lipid was PA (Sundler \& Papahadjopoulos, 1981). Here, we report that liposomes containing $5 \mathrm{~mol} \%$ PA fuse at lower $\mathrm{Ca}^{2+}$ levels and at a higher rate than PA-deficient liposomes. It should be noted that sensitivity of fusion to $\mathrm{Ca}^{2+}$ was directly related to the content of acidic phospholipids, such as PA. This could be due to enhanced anionic surface charge, which would increase the local concentrations of $\mathrm{Ca}^{2+}$ (Bentz, Düzgünes \& Nir, 1983). Also, PA could be working by enhancing phase transitions in the membranes, as it has been reported that transitions from bilayer to hexagonal $H_{I I}$ phases are critical to the fusion process (Hope, Walker \& Cullis, 1983; Cullis et al., 1985).

$\mathrm{PA}$ is produced in response to secretagogues in neutrophils (Serhan et al., 1982; Cockcroft, 1984; Agwu et al., 1989; Billah et al., 1989). The PA rise is both rapid and transient, consistent with a role for this type of membrane remodeling in fusion. In neutrophils, PA appears to result primarily from phospholipase $D$ action on PC (Cockcroft, 1984; Tou et al., 1991). Also, treatment of PC-rich liposomes with phospholipase D generates PA and enhances fusion (Park, Lee \& Kim, 1992). Hence, a strong fusion inhibitor is converted to a strong promoter in a single process. PA may also be produced from other sources, including diacylglycerol (Cockcroft, Bennett \& Gomperts, 1980), but these pathways do not appear to be involved in secretion or exocytosis. 
Remodeling to produce PA from PC may be an important mechanism for controlling the timing and location of fusion events.

In contrast to $\mathrm{PA}, \mathrm{DiC}_{8}$ had little effect on $\mathrm{Ca}^{2+}$-induced fusion of liposomes. $\mathrm{DiC}_{8}$ was selected as our model diglyceride because of its powerful ability to stimulate neutrophils (Boonen, De Koster \& Elferink, 1993; Rosenthal et al., 1993). However, we recognize that a short-chain DAG, while physiologically potent, might not be as powerful a fusogen for liposomes as longer chain varieties. Low concentrations of added $\mathrm{DiC}_{8}$ will enhance annexin-mediated fusion of PA/PE liposomes (Francis et al., 1992). Also, incorporated diacylglycerols can enhance fusion of liposomes composed of either PS/PC (Gomez-Fernandez et al., 1989) or monomethylated-PE (Siegel et al., 1989), although it dramatically increases concomitant contents leakage in either system. However, $\mathrm{DiC}_{8}$ did not enhance the fusion of $50 \%$ cholesterol complex liposomes, whether the $\mathrm{DiC}_{8}$ was incorporated or exogenously supplied. Like cholesterol, the effect of diacylglycerol seemed to depend on membrane composition.

In neutrophils, the major pathway leading to diacylglycerol production is through the hydrolysis of PA by phosphatidate phosphohydrolase (Billah et al., 1989; Della Bianca et al., 1991), rather than through the hydrolysis of phospholipids by phospholipase C. In terms of fusion, this suggests that the "window of opportunity" for fusion, provided by the production of PA from $\mathrm{PC}$, is subsequently closed by the conversion of PA to diacylglycerol.

\section{"Heterologous" Fuston}

Cell fusion typically involves the interaction of membranes from two different sources, of differing composition, and therefore having different characteristics. It was unclear whether fusion would be severely restricted by the least fusogenic membrane composition, or if fusion would proceed with the most fusogenic. Using the contents-mixing assay to measure interactions between heterologous partners, we commonly found that the fusion characteristics of heterologous fusion partners were intermediate between those of the homologous pairings. However, this was not the case when $2 \%$ PA was incorporated into only one fusion partner. For a significant PA-induced shift in fusion, $2 \%$ PA had to be present in both fusing membranes. This implies that for this form of membrane remodeling to be effective in enhancing fusion, either the PA production must occur in both interacting membranes, or else extensive remodeling must occur in one.

In this study, we evaluated only $\mathrm{Ca}^{2+}$-induced fusion, and the $\mathrm{Ca}^{2+}$ requirements were high. Other cofactors undoubtedly are involved in cell fusion (see, e.g., Düzgünes et al., 1987; Papahadjopoulos et al., 1990). For example, proteins such as annexins reduce the $\mathrm{Ca}^{2+}$ requirement for fusion (Zaks \& Creutz, 1990), interact preferentially with PA (Blackwood \& Ernst, 1990) and may play a role in cell fusion (Creutz, 1992). Also, changes in $\mathrm{Ca}^{2+}$ may be localized to a membrane region within the cell (Sawyer, Sullivan \& Mandel, 1985; Jaconi et al., 1991). Such localized concentrations of $\mathrm{Ca}^{2+}$, as well as asymmetric distributions of PA (Eastman et al., 1992) or cholesterol (Schroeder \& Nemecz, 1990) may be effective in driving cell fusion. Finally, many proteins have been implicated in fusion in other cell systems. Fusion in the Golgi system requires a protein that is sensitive to $\mathrm{N}$-ethyl maleimide (designated "NEM-sensitive fusion protein" or NSF), a Soluble NSF-Attachment Protein (SNAP), ATP, acetylCoA, "Factor B," and several "Sec" (secretion) factors, as well as specific membrane docking proteins (Clary, Griff \& Rothman, 1990; Rothman \& Orci, 1990; Bennett \& Scheller, 1993; De Camilli, 1993). In vitro secretion has been reported to be modulated by rab3A (Edwardson, MacLean \& Law, 1993; MacLean, Law \& Edwardson, 1993). Also, it has been demonstrated that similar or identical proteins, particularly SNAP's or SNAP receptors, are involved in fusion over a broad range of species and cell types (Barinaga, 1993; Bennett \& Scheller, 1993; De Camilli, 1993; Söllner et al., 1993). We expect that both changes in lipid composition and association with protein fusogens will ultimately be found to be involved in in vivo fusion. The complex liposomes that we have described here can be used in studies of fusion with these proteins in the future.

\section{References}

Agwu, D.E., McPhail, L.C., Chabot, M.C., Daniel, L.W., Wykle, R.L., McCall, C.E. 1989. Choline-linked phosphoglycerides. A source of phosphatidic acid and diglycerides in stimnlated neutrophils. J. Biol. Chem. 264:1405-1413

Barinaga, M. 1993. Secrets of secretion revealed. Science 260:487489

Bennett, M.K., Scheller, R.H. 1993. The molecular machinery for secretion is conserved from yeast to neurons. Proc. Natl. Acad. Sci. USA 90:2559-2563

Bental, M., Wilschut, J., Scholma, J., Nir, S. 1987. $\mathrm{Ca}^{2+}$-induced fusion of large unilamellar phosphatidylserine/cholesterol vesicles. Biochim. Biophys. Acta 898:239-247

Bentz, J., Düzgünes, N., Nir, S. 1983. Kinetics of divalent cation induced fusion of phosphatidylserine vesicles: correlation between fusogenic capacities and binding affinities. Biochemistry 22:33203330

Bers, D.M. 1982. A simple method for the accurate determination of free (Ca) in Ca-EGTA solutions. Am. J. Physiol. 242:C404-C408

Billah, M.M., Eckel, S., Mullmann, T.J., Egan, R.W., Siegel, M.I. 1989. Phosphatidylcholine hydrolysis by phospholipase D determines phosphatidate and diglyceride levels in chemotactic peptidestimulated human neutrophils. Involvement of phosphatidate phos- 
phohydrolase in signal transduction. J. Biol. Chem. 264:1706917077

Blackwood, R.A., Ernst, J.D. 1990. Characterization of $\mathrm{Ca}^{2+}$-dependent phospholipid binding, vesicle aggregation and membrane fusion by annexins. Biochem. J. 266:195-200

Boonen, G.J.J.C., De Koster, B.M., Elferink, J.G.R. 1993. Activation of neutrophil migration by dioctanoyl-sn-glycerol and fMet-LeuPhe is controlled by different pathways. Agents Actions $\mathbf{3 8}$ Suppl. C:C130-C132

Braun, G., Lelkes, P.I., Nir, S. 1985. Effect of cholesterol on $\mathrm{Ca}^{2+}$ induced aggregation and fusion of sonicated phosphatidylserine/cholesterol vesicles. Biochim. Biophys. Acta 812:688-694

Breisblatt, W., Ohki, S. 1976. Fusion in phospholipid spherical membranes: II. Effect of cholesterol, divalent ions and $\mathrm{pH}$. J. Membrane Biol. 29:127-146

Chaudhury, M.K., Ohki, S. 1981. Correlation between membrane expansion and temperature-induced membrane fusion. Biochim. Biophys. Acta 642:365-374

Clary, D.O., Griff, I.C., Rothman, J.E. 1990. SNAPs, a family of NSF attachment proteins involved in intracellular membrane fusion in animals and yeast. Cell 61:709-721

Cockcroft, S. 1984. $\mathrm{Ca}^{2+}$-dependent conversion of phosphatidylinositol to phosphatidate in neutrophils stimulated with fMet-LeuPhe or ionophore A23187. Biochim. Biophys. Acta 795:37-46

Cockcroft, S. 1992. G-protein-regulated phospholipases C, D and $\mathrm{A}_{2}$-mediated signalling in neutrophils. Biochim. Biophys. Acta Rev. Biomembr. 1113:135-160

Cockcroft, S., Bennett, J.P., Gomperts, B.D. 1980. Stimulus-secretion coupling in rabbit neutrophils is not mediated by phosphatidylinositol breakdown. Nature 288:275-277

Connor, J., Yatvin, M.B., Huang, L. 1984. pH-sensitive liposomes: Acid-induced liposome fusion. Proc. Natl. Acad. Sci. USA 81: $1715-1718$

Creutz, C.E. 1992. The annexins and exocytosis. Science 258:924-930

Cullis, P.R., Hope, M.J., De Kruijff, B., Verkleij, A.J., Tilcock, C.P.S. 1985. Structural properties and functional roles of phospholipids in biological membranes. In: Phospholipids and Cellular Regulation. J.F. Kuo, editor. pp. 3-59. CRC, Boca Raton, FL

De Camilli, P. 1993. Secretion: Exocytosis goes with a SNAP. $\mathrm{Na}$ ture 364:387-388

Della Bianca, V., Grzeskowiak, M., Lissandrini, D., Rossi, F. 1991. Source and role of diacylglycerol formed during phagocytosis of opsonized yeast particles and associated respiratory burst in human neutrophils. Biochem. Biophys. Res. Commun. 177:948-955

Devaux, P.F., Mathivet, L., Cribier, S., Farge, E. 1993. How lipid asymmetry can make vesicles fusion-competent by inhibition of the thermal undulations. Biochem. Soc. Trans. 21:276-280

Diez, E., Balsinde, J., Mollinedo, F. 1990. Subcellular distribution of fatty acids, phospholipids and phospholipase $A_{2}$ in human neutrophils. Biochim. Biophys. Acta Lipids Lipid Metab. 1047:83-89

Düzgünes, N., Hong, K., Baldwin, P.A., Bentz, J., Nir, S., Papahadjopoulos, D. 1987. Fusion of phospholipid vesicles induced by divalent cations and protons. Modulation by phase transitions, free fatty acids, monovalent cations, and polyamines. In: Cell Fusion. A.E. Sower, editor. pp. 241-267. Plenum, New York

Düzgünes, N., Wilschut, J., Fraley, R., Papahadjopoulos, D. 1981. Studies on the mechanism of membrane fusion: Role of headgroup composition in calcium- and magnesium-induced fusion of mixed phospholipid vesicles. Biochim. Biophys. Acta 642:182-195

Eastman, S.J., Hope, M.J., Wong, K.F., Cullis, P.R. 1992. Influence of phospholipid asymmetry on fusion between large unilamellar vesicles. Biochemistry 31:4262-4268

Eberhard, D.A., Cooper, C.L., Low, M.G., Holz, R.W. 1990. Evidence that the inositol phospholipids are necessary for exocytosis. Loss of inositol phospholipids and inhibition of secretion in permeabilized cells caused by a bacterial phospholipase $C$ and removal of ATP. Biochem. J. 268:15-25

Edwardson, J.M., MacLean, C.M., Law, G.J. 1993. Synthetic peptides of the rab3 effector domain stimulate a membrane fusion event involved in regulated exocytosis. FEBS Lett. 320:52-56

Etemadi, A.-H. 1980. Membrane asymmetry. A survey and critical appraisal of the methodology. II. Methods for assessing unequal distribution of lipids. Biochim. Biophys. Acta 604:423-475

Evans, E., Needham, D. 1986. Giant vesicle bilayers composed of mixtures of lipids, cholesterol and polypeptides. Thermomechanical and (mutual) adherence properties. Faraday Discuss. Chem. Soc. 81:267-280

Francis, J.W., Balazovich, K.J., Smolen, J.E., Margolis, D.I., Boxer, L.A. 1992. Human neutrophil annexin I promotes granule aggregation and modulates $\mathrm{Ca}^{2+}$-dependent membrane fusion. J. Clin. Invest. 90:537-544

Francis, J.W., Smolen, J.E., Balazovich, K.J., Sandborg, R.R., Boxer, L.A. 1990. Calcium-dependent fusion of the plasma membrane fraction from human neutrophils with liposomes. Biochim. Biophys. Acta 1025:1-9

Gomez-Fernandez, J.C., Aranda, F.J., Micol, V., Villalain, J., Ortiz, A. 1989. Effect of diacylglycerols on calcium-induced fusion of phosphatidylserine/phosphatidylcholine vesicles. Biochem. Soc. Trans. 17:957-960

Grubbs, R.D., Maguire, M.E. 1987. Magnesium as a regulatory cation: Criteria and evaluation. Magnesium 6:113-127

Hope, M.J., Walker, D.C., Cullis, P.R. 1983. $\mathrm{Ca}^{2+}$ and $\mathrm{pH}$ induced fusion of small unilamellar vesicles consisting of phosphatidylethanolamine and negatively charged phospholipids: a freeze fracture study. Biochem. Biophys. Res, Commun. 110:15-22

Horkovics-Kovats, S., Traub, P. 1990. Specific interaction of the intermediate filament protein vimentin and its isolated $\mathrm{N}$-terminus with negatively charged phospholipids as determined by vesicle aggregation, fusion, and leakage measurements. Biochemistry 29: $8652-8657$

Jaconi, M.E.E., Theler, J.M., Schlegel, W., Appel, R.D., Wright, S.D., Lew, P.D. 1991. Multiple elevations of cytosolic-free $\mathrm{Ca}^{2+}$ in human neutrophils: Initiation by adherence receptor of the integrin family. J. Cell Biol. 112:1249-1257

Kielian, M.C., Helenius, A. 1984. Role of cholesterol in fusion of Semliki Forest virus with membranes. J. Virol. 52:281-283

MacLean, C.M., Law, G.J., Edwardson, J.M. 1993. Stimulation of exocytotic membrane fusion by modified peptides of the rab3 effector domain: Re-evaluation of the role of rab3 in regulated exocytosis. Biochem. J. 294:325-328

Mason, R.J., Stossel, T.P., Vaughan, M. 1972. Lipids of alveolar macrophages, polymorphonuclear leukocytes, and their phagocytic vesicles. J. Clin. Invest. 51:2399-2407

Meers, P., Ernst, J.D., Düzgünes, N., Hong, K.L., Fedor, J., Goldstein, L.M., Papahadjopoulos, D. 1987. Synexin-like proteins from human polymorphonuclear leukocytes. Identification and characterization of granule-aggregating and membrane-fusing activities. $J$. Biol. Chem. 262:7850-7858

Morrison, W.R. 1964. A fast, simple and reliable method for the microdetermination of phosphorus in biological materials. Anal. Biochem. 7:218-224

Mueller, H.W., O'Flaherty, J.T., Greene, D.G., Samuel, M.P., Wykle, R.L. 1984. 1-O-Alkyl-linked glycerophospholipids of human neutrophils: distribution or arachidonate and other acyl residues in the ether-linked and diacyl species. J. Lipid Res. 25:383-388

Nachman, R., Hirsch, J.G., Baggiolini, M. 1972. Studies on isolated membrane of azurophil and specific granules from rabbit polymorphonuclear leukocytes. J. Cell Biol. 54:133-140 
Nieva, J.L., Goni, F.M., Alonso, A. 1989. Liposome fusion catalytically induced by phospholipase C. Biochemistry 28:7364-7367

Papahadjopoulos, D., Poste, G., Schaeffer, B.E., Vail, W.J. 1974. Membrane fusion and molecular segregation in phospholipid vesicles. Biochim. Biophys. Acta 352:10-28

Papahadjopoulos, D., Nir, S., Düzgünes, N. 1990. Molecular mechanisms of calcium-induced membrane fusion. J. Bioenerg. Biomemb. 22:157-179

Park, J.-B., Lee, T.-H., Kim, H. 1992. Fusion of phospholipid vesicles induced by phospholipase $\mathrm{D}$ in the presence of calcium ion. Biochem. Int. 27:417-422

Raja, K.B., Leach, P.M., Peters, G.P., McCarthy, D., Peters, T.J. 1982. The concentration and subcellular localisation of zinc, magnesium and calcium in human polymorphonuclear leukocytes. Clin. Chim. Acta 123:19-26

Rand, R.P., Parsegian, V.A. 1986. Mimicry and mechanism in phospholipid models of membrane fusion. Annu. Rev. Physiol. 48:201212

Rosenthal, M.D., Lattanzio, K.S., Franson, R.C. 1993. 1,3-Dioctanoylglycerol modulates arachidonate mobilization in human neutrophils and its inhibition by PGBx: evidence of a protein-kinase-C-independent role for diacylglycerols in signal transduction. Biochim. Biophys. Acta Mol. Cell Res. 1177:79-86

Rothman, J.E., Orci, L. 1990. Mavement of proteins through the Golgi stack: a molecular dissection of vesicular transport. FASEB J. 4:1460-1468

Santini, M.T., Indovina, P.L., Cantafora, A., Blotta, I. 1990. The cesium-induced delay in myoblast membrane fusion is accompanied by changes in isolated membrane lipids. Biochim. Biophys. Acta 1023:298-304

Sawyer, D.W., Sullivan, J.A., Mandell, G.L. 1985. Intracellular free calcium localization in neutrophils during phagocytosis. Science 230:663-666

Schroeder, F., Nemecz, G. 1990. Transmembrane cholesterol distribution. In: Advances in Cholesterol Research. M. Esfahani and J.B. Swaney, editors. pp. 47-87. Telford, Caldwell, NJ

Serhan, C.N., Broekman, M.J., Korchak, H.M., Marcus, A.J., Weissmann, G. 1982. Endogenous phospholipid metabolism in stimulated neutrophils. Differential activation by FMLP and PMA. Biochem. Biophys. Res. Commun. 107:951-958

Sessions, A., Horwitz, A.G. 1983. Differentiation-related differences in the plasma membrane phospholipid asymmetry of myogenic and fibrogenic cells. Biochim. Biophys. Acta 728:103-111

Siegel, D.P., Banschbach, J., Alford, D., Ellens, H., Lis, L.J., Quinn, P.J., Yeagle, P.L., Bentz, J. 1989. Physiological levels of diacyl- glycerols in phospholipid membranes induce membrane fusion and stabilize inverted phases. Biochemistry 28:3703-3709

Smolen, J.E. 1992. Neutrophil signal transduction: Calcium, kinases, and fusion. J. Lab. Clin. Med. 120:527-532

Smolen, J.E., Sandborg, R.R. $1990 . \mathrm{Ca}^{2+}$-induced secretion by electropermeabilized human neutrophils. The roles of $\mathrm{Ca}^{2+}$, nucleotides and protein kinase C. Biochim. Biophys. Acta 1052:133142

Smolen, J.E., Shohet, S.B. 1974. Remodeling of granulocyte membrane fatty acids during phagocytosis. J. Clin. Invest. 53:726-734

Söllner, T., Whiteheart, S.W., Brunner, M., Erdjument-Bromage, H., Geromanos, S., Tempst, P., Rothman, J.E. 1993. SNAP receptors implicated in vesicle targeting and fusion. Nature 362:318-324

Stamatotos, L., Silvius, J.R. 1987. Effects of cholesterol on the divalent cation-mediated interactions of vesicles containing amino and choline phospholipids. Biochim. Biophys. Acta 905:81-90

Sundler, R., Düzgünes, N., Papahadjopoulos, D. 1981. Control of membrane fusion by phospholipid head groups. II. The role of phosphatidylethanolamine in mixtures with phosphatidate and phosphatidylinositol. Biochim. Biophys. Acta 649:751-758

Sundler, R., Papahadjopoulos, D. 1981. Control of membrane fusion by phospholipid head groups. I. Phosphatidate/phosphatidylinositol specificity. Biochim. Biophys. Acta 649:743-750

Tou, J., Jeter, J.R., Jr., Dola, C.P., Venkatesh, S. 1991. Accumulation of phosphatidic acid mass and increased de novo synthesis of glycerolipids in platelet-activating-factor-activated human neutrophils. Biochem. J. 280:625-629

Uster, P.S., Deamer, D.W. 1981. Fusion competence of phosphatidylserine-containing liposomes quantitatively measured by a fluorescence resonance energy transfer assay. Arch. Biochem. Biophys. 209:385-395

Verhoven, B., Schlegel, R.A., Williamson, P. 1992. Rapid loss and restoration of lipid asymmetry by different pathways in resealed erythrocyte ghosts. Biochim. Biophys. Acta Bio-Membr. 1104:1523

Werb, Z., Cohn, Z.A. 1972. Plasma membrane synthesis in the macrophage following phagocytosis of polystyrene latex particles. J. Biol. Chem. 247:2439-2446

Woodin, A.M., Wieneke, A.A. 1966. Composition and properties of a cell-membrane fraction from the polymorphonuclear leucocyte. Biochem, J. 99:493-500

Zaks, W.J., Creutz, C.E. 1990. Evaluation of the annexins as potential mediators of membrane fusion in exocytosis. J. Bioenerg. Biomemb. 22:97-120 